\title{
The Vela-Puppis open clusters Pismis 8 and Pismis $13^{\star}, \star \star$
}

\author{
E. E. Giorgi ${ }^{1}$, G. Baume ${ }^{1,2}$, G. Solivella ${ }^{1}$, and R. A. Vázquez ${ }^{1}$ \\ ${ }^{1}$ Facultad de Ciencias Astronómicas y Geofísicas, UNLP, and IALP (UNLP - CONICET), Paseo del Bosque s/n, 1900, \\ La Plata, Argentina \\ e-mail: egiorgi@fcaglp.unlp.edu.ar \\ 2 Dipartimento di Astronomia, Università di Padova, Vicolo Osservatorio 2, 35122 Padova, Italy
}

Received 12 April 2004 / Accepted 31 October 2004

\begin{abstract}
CCD UBVI imaging photometry was carried out in the fields of the open clusters Pismis 8 and Pismis 13, located in the Vela-Puppis region in our Galaxy. $M K$ spectral types have also been determined for a number of stars located in the fields of these two clusters which were used to secure membership among the brightest stars. Since our photometry goes to a fainter limit than previous studies we could provide better reddening, distance and age determinations. Both clusters are located close to the edge of the local arm in the third quadrant. Pismis 8 is a cluster about 5-7 My old located at $2000 \mathrm{pc}$ from the Sun, while Pismis 13 was found at $2750 \mathrm{pc}$ with a probable age of about $100 \mathrm{My}$. The estimate of the slopes of the mass functions in both cases yielded $x \approx 1.7$ and $x \approx 2.1$ for Pismis 8 and 13 respectively.
\end{abstract}

Key words. Galaxy: open clusters and associations: individual: Pismis 8, Pismis 13 - stars: luminosity function, mass function - stars: Hertzsprung-Russell (H-R) and C-M diagrams

\section{Introduction}

The Vela-Puppis region is a remarkable feature of the spiral structure of our Galaxy close to the frontier between the third and fourth quadrants which extends from $240^{\circ}$ to $280^{\circ}$ in galactic longitude. In this place, windows in the interstellar absorption allow a deep view of outlying parts of the galactic disk. Many clusters have been found in this zone but only a few of them have been investigated photometrically. This prevents us from learning about the way the spiral structure of our Galaxy develops and from better knowing the stellar content in Vela-Puppis. The situation is now changing thanks to the systematic efforts made by Moitinho (2001), Giorgi et al. (2002), Baume et al. (2003) and Carraro \& Munari (2004) among others.

What makes the third quadrant most important is that it contains several distant clusters through which information on the kinematics and evolutionary status of the stellar population in the outermost parts of the galactic disk can be extracted; it also contains nearby clusters by which the local arm structure can be outlined precisely.

Our ongoing program on open clusters was designed to study small and sparse open clusters to achieve a better

^ Based on observations collected at the University of Toronto Southern Observatory, Las Campanas, Chile, and the Complejo Astronómico El Leoncito, San Juan, Argentina.

$\star \star$ Table 3 is only available in electronic form at the CDS via anonymous ftp to cdsarc.u-strasbg.fr $(130.79 .128 .5)$ or via http://cdsweb.u-strasbg.fr/cgi-bin/qcat?]/A+A/432/491 determination of their fundamental parameters. On this occasion, we decided to undertake CCD UBVI photometry plus spectroscopy in the Vela-Puppis spur clusters Pismis $8\left(\alpha_{2000}=\right.$ $\left.08^{\mathrm{h}} 41^{\mathrm{m}} 36^{\mathrm{s}}, \delta_{2000}=-46^{\circ} 16^{\prime} 00^{\prime \prime}\right)$ and Pismis $13\left(\alpha_{2000}=\right.$ $\left.09^{\mathrm{h}} 22^{\mathrm{m}} 07^{\mathrm{s}}, \delta_{2000}=-51^{\circ} 06^{\prime} 00^{\prime \prime}\right)$.

The aim of this work is therefore to present a homogeneous photometric sample of two star clusters in this portion of the Galaxy, to reassess their parameters and to examine the lower main sequences as deep as possible. An additional effort will be made to determine their mass functions to contribute to the construction of a new and extended data base to compare characteristics of the objects in this part of the Galaxy with those from previous studies made in other galactic zones. In Sect. 2 we briefly present the observations and data reduction procedures. In Sect. 3 we describe the methods used for the determination of the basic cluster parameters, membership, reddening, distance and age. In Sect. 4 we show individual results and in Sect. 5 we give a brief discussion and the concluding remarks.

\section{Observations}

\section{1. $C C D$ photometry}

CCD imaging photometry in the UBVI Cousins system was carried out in February 1996. Data were obtained with the $60-\mathrm{cm}$ telescope of the University of Toronto Southern Observatory at Las Campanas Observatory, Chile, and a PM $512 \times$ 512 METHACROME-II UV coated CCD. The scale of the chip is $0.45^{\prime \prime} /$ pixel, covering thus a field of $4^{\prime}$ on a side. 

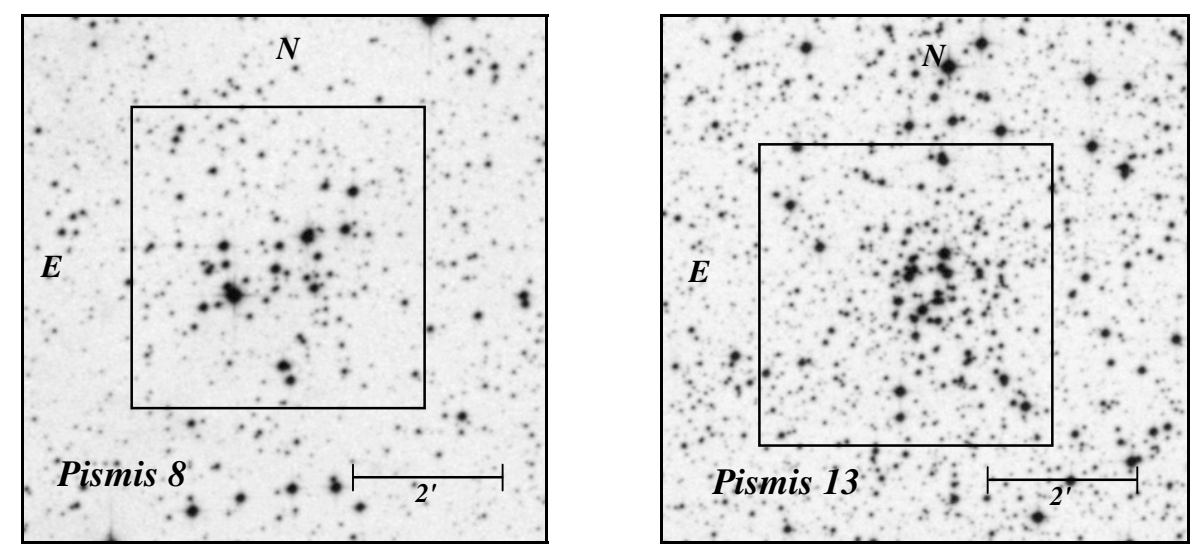

Fig. 1. The finding chart of Pismis 8 and Pismis 13 adapted from DSS plates. The squares show the area covered in our survey. The scale is shown at the bottom. North and East are indicated.

Table 1. Journal of science-frame observations and exposure times (s). The number of averaged frames is given in parentheses.

\begin{tabular}{llrrrr}
\hline \hline Cluster & Exposure time & $U$ & $B$ & $V$ & $I$ \\
\hline Pismis 13 & short & & 200 & 100 & 20 \\
$23 / 02 / 1996$ & long & $1000(2)$ & 1000 & 600 & 130 \\
\hline Pismis 8 & short & 200 & 30 & 10 & 2 \\
$25 / 02 / 1996$ & medium & & 200 & 100 & 20 \\
& long & $1000(2)$ & 1000 & 600 & 130 \\
\hline
\end{tabular}

The nights were photometric with seeing values ranging from $1.2^{\prime \prime}$ to $1.4^{\prime \prime}$. Pismis 8 was observed on February 23 and Pismis 13 on February 25. Details of exposure times and the number of observations can be found in Table 1. The charts of the two clusters showing the positioning of the respective frames are shown in Fig. 1.

Instrumental magnitudes were obtained by point spread function (PSF) fitting using the DAOPHOT (Stetson 1987) package within IRAF $^{1}$. These values were transformed into the standard system through a set of more than thirty stars observed in the open clusters NGC 5606 (Vázquez et al. 1994) and Trumpler 18 (Vázquez \& Feinstein 1990). The extinction coefficients were taken from Grotues \& Gocherman (1992) and the transformation equations to the standard system were of the form:

$u=U+u_{1}+u_{2} \times X+u_{3} \times(U-B)$

$b=B+b_{1}+b_{2} \times X+b_{3} \times(B-V)$

$v=V+v_{1}+v_{2} \times X+v_{3} \times(B-V)$

$i=I+i_{1}+i_{2} \times X+i_{3} \times(V-I)$

where $u_{2}, b_{2}, v_{2}$ and $i_{2}$ are the extinction coefficients for the $U B V I$ bands respectively, $X$ the air masses for each exposure and $u_{1}, b_{1}, v_{1}, i_{1}, u_{3}, b_{3}, v_{3}$, and $i_{3}$ the fitting constants values given in Table 2 .

Table 3 (available in electronic format at CDS) contains the $x-y$ star positions and photometric measures in Pismis 8

\footnotetext{
${ }^{1}$ IRAF is distributed by NOAO, which are operated by AURA under cooperative agreement with the NSF.
}

Table 2. Values and errors of the fitting constants.

\begin{tabular}{crr}
\hline \hline Constants & $02 / 23 / 1996$ & $02 / 25 / 1996$ \\
\hline$u_{1}$ & $7.24 \pm 0.02$ & $7.36 \pm 0.01$ \\
$u_{2}$ & 0.514 & 0.514 \\
$u_{3}$ & $-0.11 \pm 0.03$ & $-0.15 \pm 0.05$ \\
$b_{1}$ & $5.79 \pm 0.01$ & $5.87 \pm 0.01$ \\
$b_{2}$ & 0.314 & 0.314 \\
$b_{3}$ & $-0.12 \pm 0.02$ & $-0.13 \pm 0.02$ \\
$v_{1}$ & $5.43 \pm 0.02$ & $5.50 \pm 0.06$ \\
$v_{2}$ & 0.208 & 0.208 \\
$v_{3}$ & $-0.07 \pm 0.02$ & $-0.05 \pm 0.10$ \\
$i_{1}$ & $5.14 \pm 0.02$ & $5.18 \pm 0.01$ \\
$i_{2}$ & 0.004 & 0.004 \\
$i_{3}$ & $-0.07 \pm 0.02$ & $-0.04 \pm 0.02$ \\
\hline
\end{tabular}

where $V, B-V$ and $V-I$ were obtained for 104 stars and $U-B$ for 60 stars. For Pismis $13, V$ and $B-V$ were derived for 260 stars, $U-B$ for 113 stars and $V-I$ for 255 stars. Mean differences of our photometry to two previous data sets were computed in magnitudes and colors as shown in Table 4 in the sense "our photometry minus other authors" for a number of common stars indicated in Col. 5. Some discrepancies were found with the measures of Pismis 8 made by FitzGerald et al. (1979a) but they performed photoelectric photometry for 9 cluster stars one measurement alone on averge - and photographic photometry for the rest of them. As they claim that the quality of the nights was not good this may explain the differences detected between both sets. Regarding the comparison with the Clariá (1979) photoelectric data of Pismis 13, we only found irrelevant differences in the $B-V$ index. During writing of this paper, a $B V I$ CCD photometric study including NGC 2866 (another designation for Pismis 13) was published by Carraro \& Munari (2004). Here, we briefly compare their result with ours. The $\Delta V$ and $\Delta(B-V)$ differences between Carraro \& Munari and Clariá data are $0.058 \pm 0.114$ and $-0.028 \pm 0.039$, quite similar to, and with the same sign as the differences between our data and those of Clariá. 

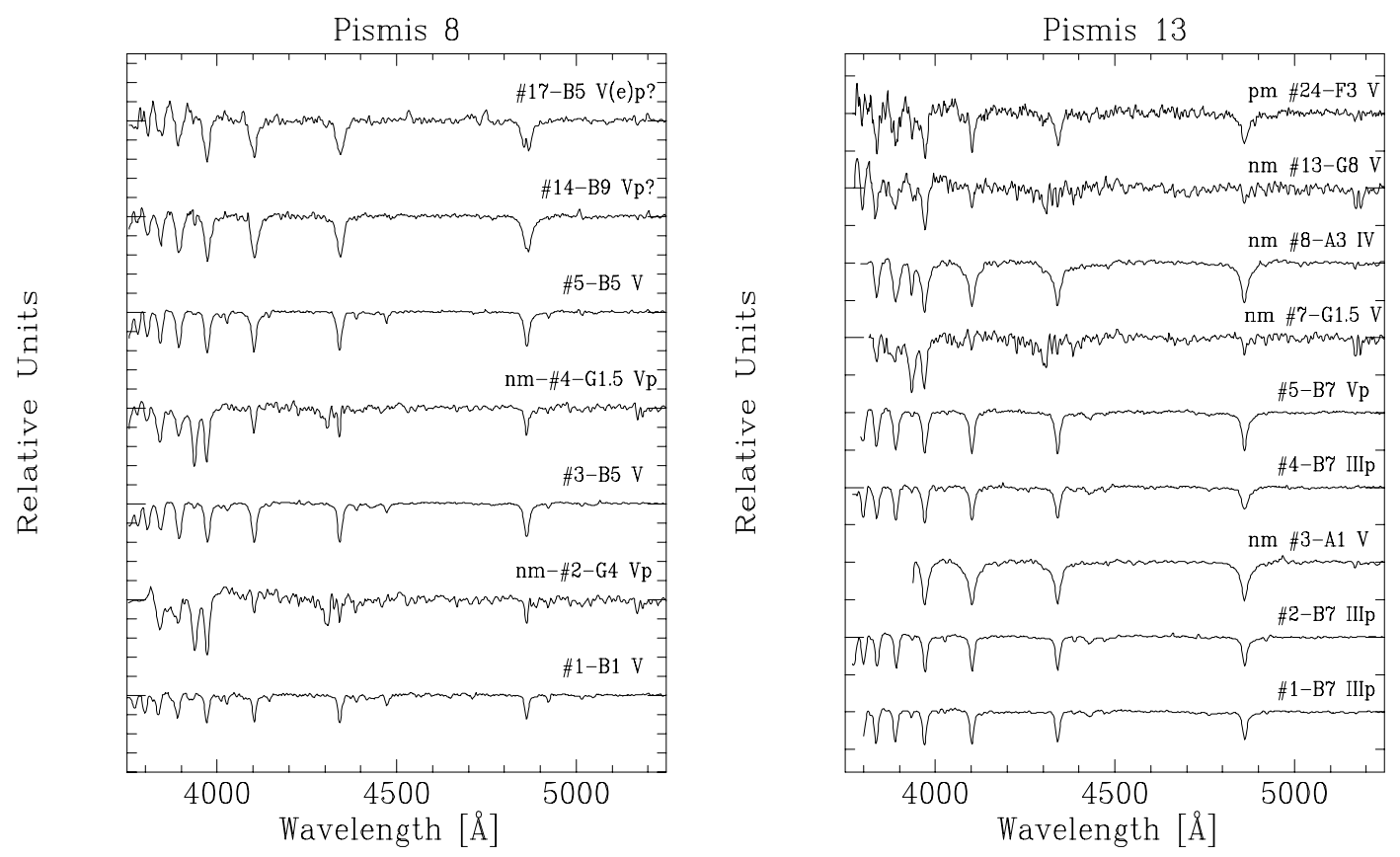

Fig. 2. The spectra of 7 and 9 stars in Pismis 8 and 13 respectively grouped by our classification given in Table 5. Non-members (nm) and probable members $(\mathrm{pm})$ are indicated.

Table 4. Comparison with previous photometries.

\begin{tabular}{lrrrrll}
\hline \hline Cluster & $\Delta(V)$ & $\Delta(B-V)$ & $\Delta(U-B)$ & $N$ & Data-type & Ref. \\
\hline Pismis 8 & $0.08 \pm 0.14$ & $0.05 \pm 0.15$ & $-0.09 \pm 0.18$ & 17 & pe \& pg & FitzGerald et al. (1979a) \\
Pismis 13 & $0.01 \pm 0.11$ & $-0.05 \pm 0.04$ & $0.01 \pm 0.04$ & 23 & pe & Clariá (1979) \\
\hline
\end{tabular}

Note for data-type: pe = photoelectric; pg =photographic

\subsection{Spectroscopy}

Spectral data were collected at the $215-\mathrm{cm}$ telescope of CASLEO (Argentina) during three observing runs. The first one was in March 12-15, 2002 when seven stars in the field of Pismis 8 were observed and the second and third ones in February 22-24, 2003, and March 12-13, 2004, when nine stars were observed in the field of Pismis 13. Observations were carried out with a REOSC-DS Cassegrain spectrograph equipped with a Tek $1024 \times 1024$ detector. The grating was centered at $8^{\circ} 30^{\prime}$ for a full spectral range coverage from 3900 to $5500 \AA$ in wavelength over the classical MK region. The dispersion was $2.5 \AA /$ pixel and the resolution of $\approx 1800$. At least two spectra were taken for each star with exposures times ranging from 20 to 40 min depending on the star magnitude and color. This way, a safe removal of any radiation event (cosmic rays) could be done. Comparison lamp spectra were also exposed before and after each star exposure. Spectra were all reduced using available IRAF routines.

Figure 2 shows the spectra obtained in each cluster together with their classification. For an appropriate location of the stars into the MK classification scheme, as shown in Table 5, we used spectra of MK standard stars taken using the same configuration at CASLEO, the Digital Spectra Classification
Atlas $^{2}$ and the MK Standard Stars online ${ }^{3}$. The spectral classification was performed using intensity ratios of CaII, HeI, $\mathrm{MgI}$ and $\mathrm{H}$. In particular, intensity ratios of HeI 4471 and MgI $4481 \AA$ were carefully examined before the final MK spectral type was assigned. Some of the stars present in Pismis 13 show spectral peculiarities; since, as expected, their colours have discrepancies to the corresponding spectral type, they were classified as peculiar stars following the criteria given by Garrison (1973).

\section{Data analysis}

\subsection{Cluster sizes}

As a first step, we estimated the angular sizes of both clusters by means of star counts. Since our CCD fields do not entirely cover the clusters and their outskirts, we performed star counts on DSS plates centered on the positions given by Dias et al. (2002). Although open clusters cannot be assumed spherical (Tapia et al. 2003) the point of the highest concentration of stars is easily revealed by star counts. Therefore, we first detected all

\footnotetext{
2 R. O. Gray;

http://nedwww.ipac.caltech.edu/level5/Gray

${ }^{3}$ http://stellar.phys.appstate.edu/Standards
} 
Table 5. MK Spectral classification, intrinsic indices and absolute magnitudes of stars in Pismis 8 and Pismis 13.

\begin{tabular}{|c|c|c|c|c|c|c|c|c|c|c|}
\hline & Pismis 8 & & & & & & & & & \\
\hline Star & Spectral type & V & $B-V$ & $U-B$ & $E_{B-V}$ & $E_{U-B}$ & $(B-V)_{0}$ & $(U-B)_{0}$ & $M_{V}$ & Remarks \\
\hline $1^{1}$ & $\mathrm{~B} 1 \mathrm{~V}$ & 10.55 & 0.57 & -0.36 & 0.85 & 0.65 & -0.28 & -1.01 & -3.58 & $\mathrm{~m}$ \\
\hline 2 & $\mathrm{G} 4 \mathrm{Vp}^{3}$ & 12.30 & 1.17 & 0.55 & & & & & & $\mathrm{~nm}$ \\
\hline $3^{1}$ & B5 V & 12.78 & 0.58 & -0.08 & 0.77 & 0.58 & -0.19 & -0.66 & -1.11 & $\mathrm{~m}$ \\
\hline 4 & $\mathrm{G} 1.5 \mathrm{Vp}^{4}$ & 12.94 & 0.56 & -0.05 & & & & & & $\mathrm{~nm}$ \\
\hline $5^{1}$ & B5 V & 13.03 & 0.62 & -0.07 & 0.82 & 0.63 & -0.20 & -0.70 & -1.01 & $\mathrm{~m}$ \\
\hline $14^{1}$ & $\mathrm{~B} 9 \mathrm{Vp} ?^{5}$ & 14.23 & 0.72 & 0.39 & 0.80 & 0.61 & -0.08 & -0.22 & +0.25 & $\mathrm{~m}$ \\
\hline \multirow[t]{2}{*}{$17^{1}$} & B5 V(e)p? ${ }^{6}$ & 14.52 & 0.64 & 0.36 & 0.71 & 0.53 & -0.07 & -0.17 & +0.82 & $\mathrm{~m}$ \\
\hline & Pismis 13 & & & & & & & & & \\
\hline Star & Spectral type & V & $B-V$ & $U-B$ & $E_{B-V}$ & $E_{U-B}$ & $(B-V)_{0}$ & $(U-B)_{0}$ & $M_{V}$ & Remarks \\
\hline $1^{1}$ & B7 IIIp $^{7}$ & 12.09 & 0.49 & 0.03 & 0.62 & 0.47 & -0.13 & -0.44 & -2.04 & $\mathrm{~m}$ \\
\hline $2^{1}$ & B7 IIIp ${ }^{7}$ & 12.65 & 0.42 & -0.08 & 0.58 & 0.43 & -0.16 & -0.51 & -1.34 & $\mathrm{~m}$ \\
\hline $3^{2}$ & $\mathrm{~A} 1 \mathrm{~V}^{8}$ & 12.74 & 0.37 & 0.29 & 0.36 & 0.27 & 0.01 & 0.02 & +1.00 & $\mathrm{~nm}$ \\
\hline $4^{1}$ & B7 IIIp ${ }^{7}$ & 12.79 & 0.50 & 0.04 & 0.63 & 0.48 & -0.13 & -0.44 & -1.37 & $\mathrm{~m}$ \\
\hline $5^{1}$ & $\mathrm{~B} 7 \mathrm{Vp}{ }^{9}$ & 12.88 & 0.60 & 0.11 & 0.74 & 0.56 & -0.14 & -0.45 & -1.61 & $\mathrm{~m}$ \\
\hline $7^{2}$ & G1.5 V & 13.17 & 0.69 & 0.31 & 0.09 & 0.21 & 0.60 & 0.10 & +4.50 & $\mathrm{~nm}$ \\
\hline 8 & A3 IV & 13.38 & 0.47 & 0.35 & & & & & & $\mathrm{~nm}$ \\
\hline $13^{2}$ & G8 V & 13.85 & 0.76 & 0.49 & 0.02 & 0.19 & 0.74 & 0.30 & +5.50 & $\mathrm{~nm}$ \\
\hline $24^{2}$ & F3 $\mathrm{V}^{8}$ & 14.81 & 0.68 & 0.25 & 0.28 & 0.25 & 0.40 & 0.00 & +3.50 & $\mathrm{~nm}$ \\
\hline
\end{tabular}

Remarks: $\mathrm{m}=$ member, $\mathrm{nm}=$ non-member.

Note 1: Color excesses, intrinsic colors and absolute magnitudes from:

$\left({ }^{1}\right)$ Photometry;

Note 2:

(2) spectral type (Schmidt-Kaler 1982).

$\left(^{3}\right) \mathrm{KCa}$ II - F0 V, H - G4 V, Mgb - G0 V;

$\left({ }^{4}\right) \mathrm{KCa}$ II $-\mathrm{G} 1.5 \mathrm{~V}, \mathrm{H}-\mathrm{G} 1.5 \mathrm{~V}, \mathrm{Mgb}-\mathrm{G} 0 \mathrm{~V}$;

$\left({ }^{5}\right) \mathrm{KCa}$ II $-\mathrm{B} 9 \mathrm{~V}, \mathrm{H}-\mathrm{B} 9 \mathrm{~V}, \mathrm{HeI}-\mathrm{A} 0 \mathrm{~V}$;

$\left({ }^{6}\right) \mathrm{KCa}$ II - B9 V, H - B5 V, H $\beta$ weak emission;

( $\left.{ }^{7}\right) \mathrm{KCa}$ II - B2 V, H - B2 V, He I (4026) - B8 V and He I (4471) /Mg II (4481) - B7 III;

$\left.{ }^{8}\right) \mathrm{KCa}$ II $-\mathrm{A} 1 \mathrm{~V}$;

$\left({ }^{9}\right) \mathrm{KCa}$ II - B2 V, H - B7 V, He I (4026) - B9 V.

stars in the plates above an adequate threshold and computed their DSS magnitudes with DAOPHOT. The counts were then made in concentric annuli and divided by the area of each annulus. Some saturated stars were lost during the reduction process but this has no influence on the final size of the clusters. As the estimated photometric limits of DSS plates go down to $V \sim 17$ the cluster radii obtained in this work refer mainly to all stars above that magnitude limit. A similar procedure was done using 2MASS data. The star density variations as a function of the cluster center distances (in arcminutes) are shown in Fig. 3 together with the average background star level. In each case we adopted as cluster radius, given in Table 8 , the distance at which the density profile merges with the background density. In both cases we found an excellent agreement of the cluster sizes derived from the DSS or 2MASS data.

\subsection{Membership and reddenings}

Figures 4-7 show the TCDs and CMDs - the two-color and color-magnitude diagrams respectively - of the two clusters.
The membership estimation in each case is based primarily on photometric arguments through a careful inspection of the position of each star in the TCDs and CMDs. That is only valid for the brightest stars where the contamination by field interlopers has little influence. Earlier discussions in the literature on the determinations of membership were taken into account as well.

Spectral types, on the other hand, allowed a refinement of cluster memberships. On spectroscopic grounds we mention the presence in the spectra of all cluster members (Pismis 8 and Pismis 13) of the diffuse interstellar band $4428 \AA$ (Snow et al. 2002), a noticeable feature in the spectra of distant $O$ and B type stars. This band is absent in non member stars, in agreement with their foreground nature (see Fig. 2).

Individual intrinsic colors of bright cluster members that admit unique reddening solutions in the TCD were obtained through the relations $E_{U-B} / E_{B-V}=0.72+0.05 \times E_{B-V}$ and $(U-B)_{0}=3.69 \times(B-V)_{0}+0.03$ (Vázquez \& Feinstein $1991)$ that are entirely valid in this region according to the analysis made by Moitinho (2001). The mean color excesses 

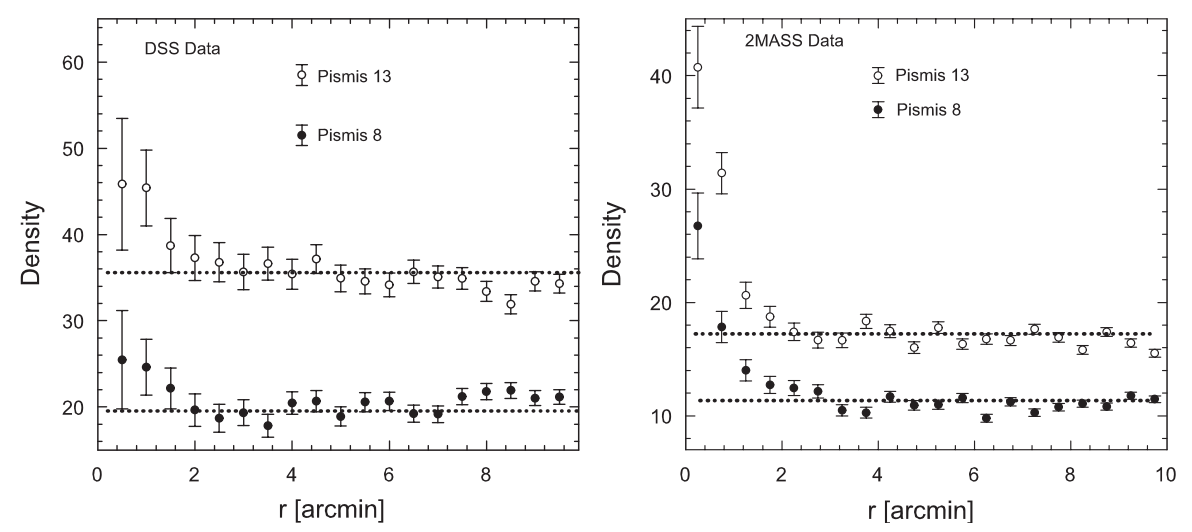

Fig. 3. The star density profiles of Pismis 8 (filled circles) and Pismis 13 (open circles) derived from star counts in DSS images (left panel) and from the 2MASS data (right panel) as a function of the cluster center distance $(r)$. The dotted lines represent the average background star density while bars are the $\sqrt{N}$ of the counts.

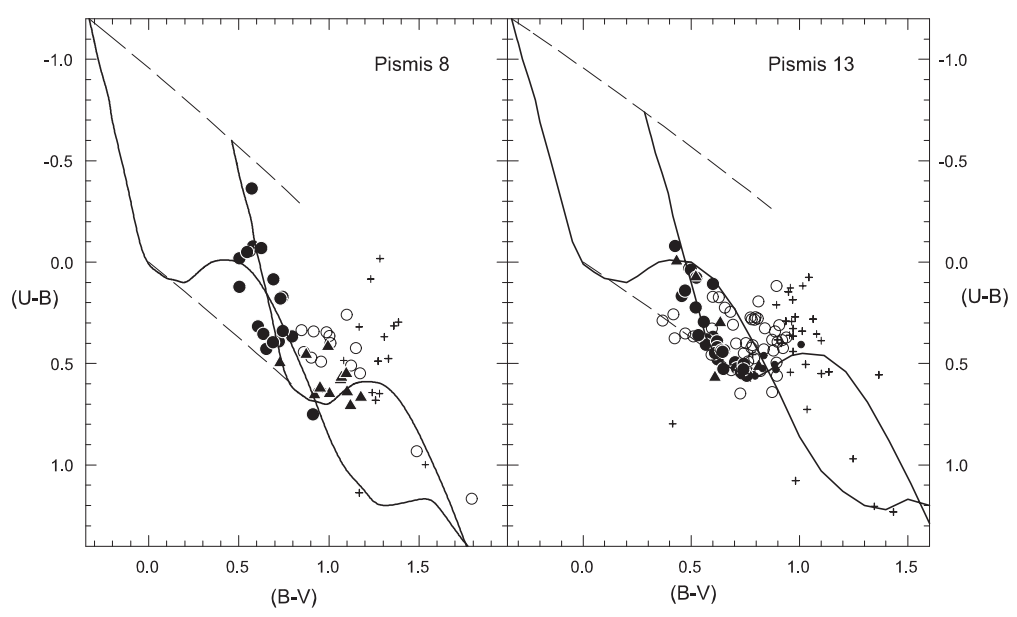

Fig. 4. The color-color diagrams of the two clusters. Symbols are as follow: large black circles for likely members; open circles for non-members; filled triangles for probable members; plus signs denote stars with photometric errors larger than 0.1 and small filled dots represent stars for which no possible membership assignment was possible. The luminosity class V sequence of Schmidt-Kaler (1982), in its normal location, and shifted to fit the cluster stars, is also shown. The dashed lines indicate the path of the reddening for stars of O and A0-types.

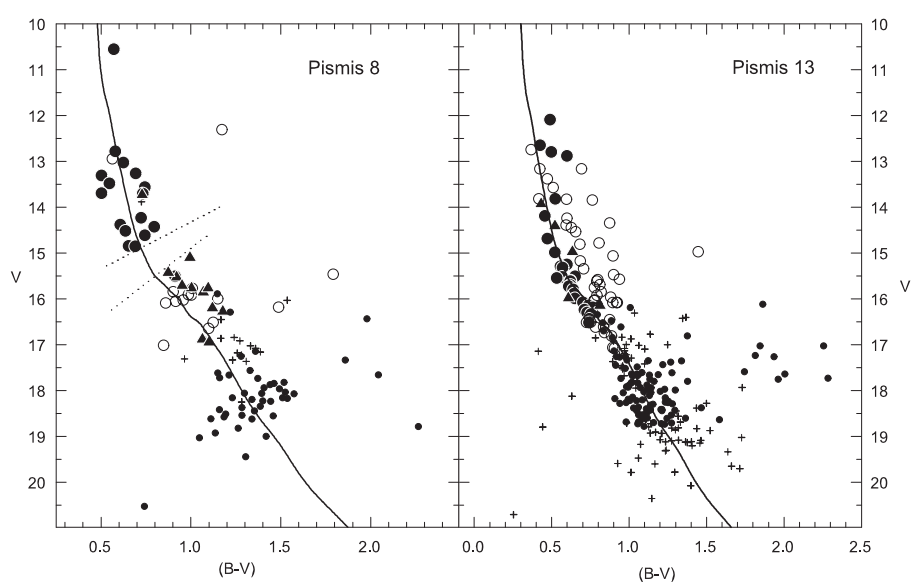

Fig. 5. The $V / B-V$ color-magnitude diagrams. Symbols as in Fig. 4. The Schmidt-Kaler ZAMS (1982) is shown fitted at apparent distance moduli of 13.95 for Pismis 8 and 14.09 for Pismis 13. Dotted lines in Pismis 8 figure shows the upper and lower limit of the "gap" discussed in the text.

of the two clusters indicated in Table 8 were used later on to obtain intrinsic colors for probable members for which no a reliable individual reddening estimation was possible. As a final step we assessed the extinction law value towards each cluster by analyzing the star distribution in the $(B-V)$ vs. $(V-I)$ color-color diagrams shown in Fig. 8. 


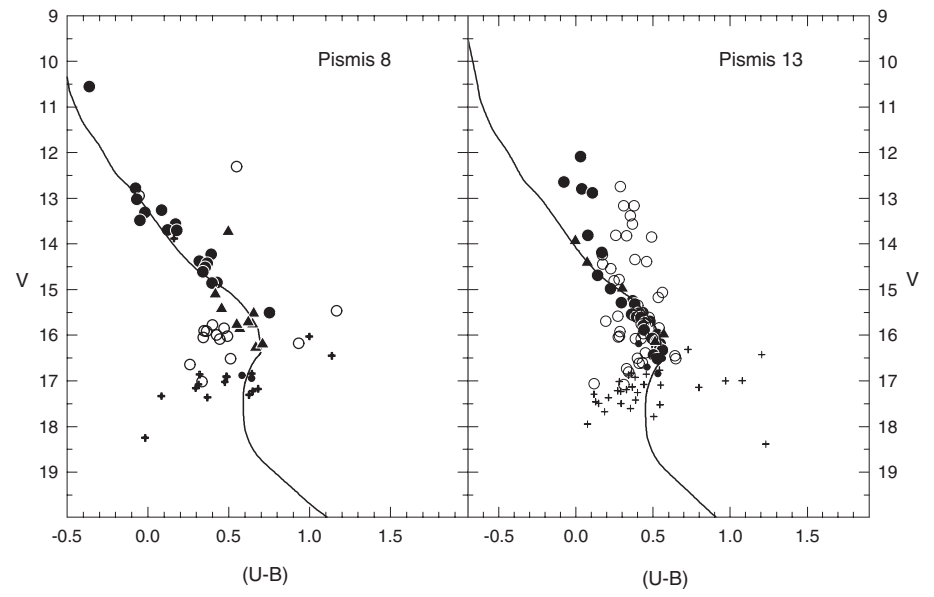

Fig. 6. The $V / U-B$ color-magnitude diagrams. Symbols as in Fig. 4. The ZAMS fittings are as in Fig. 5.

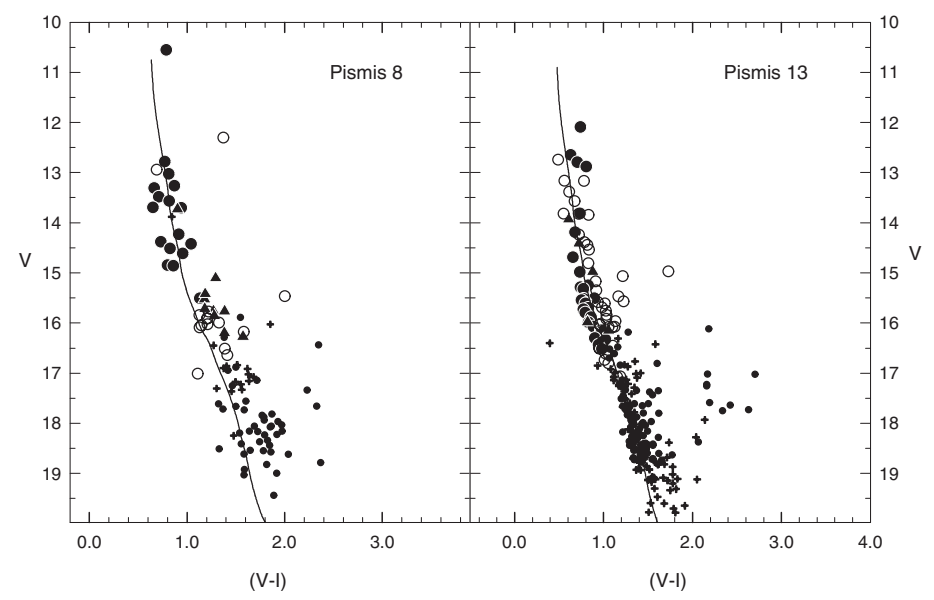

Fig. 7. The $V / V-I$ color-magnitude diagrams. Symbols as in Fig. 4. The mean locus of the reddened main sequence from Schmidt-Kaler (1982) and Cousins (1978) is shown adapted to distance values of Fig. 5.

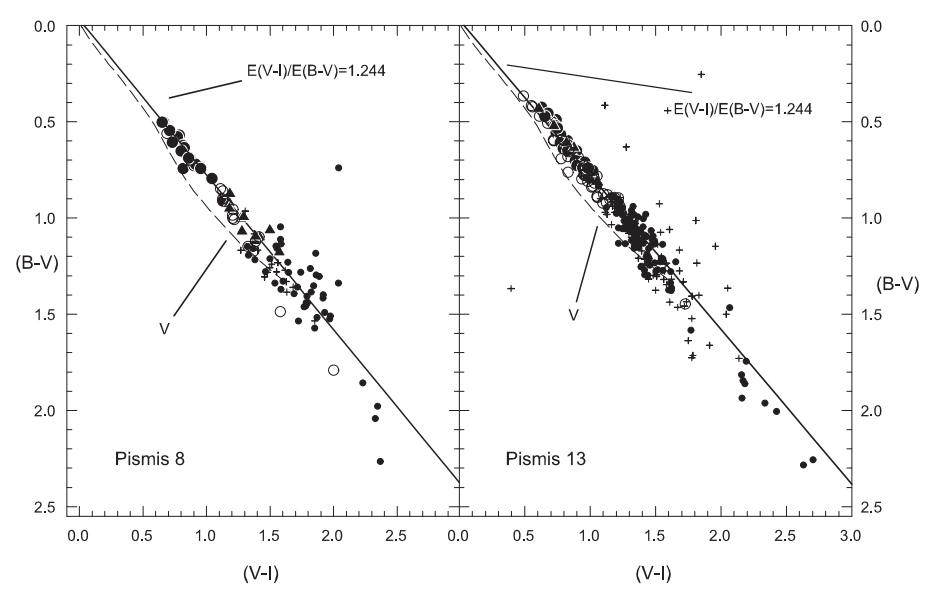

Fig. 8. The $B-V / V-I$ color-color diagrams. Symbols as in Fig. 4. The continuous line is the relation found by Dean et al. (1978) for stars affected by a normal reddening law. We also show with dashed lines the locus occupied by un-reddened stars of luminosity $V$ from Cousins (1978).

\subsection{Distances}

The distance modulus of each cluster was obtained fitting Schmidt-Kaler's (1982) ZAMS to the respective de-reddened main sequence. Distance moduli are averages of the best fits in both the $V_{0}$ vs. $(B-V)_{0}$ and $V_{0}$ vs. $(U-B)_{0}$ planes and the quoted errors of the moduli (always $\leq 0.3$ ) were all estimated by eye inspection. The final absorption-free CMDs are shown in Fig. 9 together with the location of the ZAMS. 


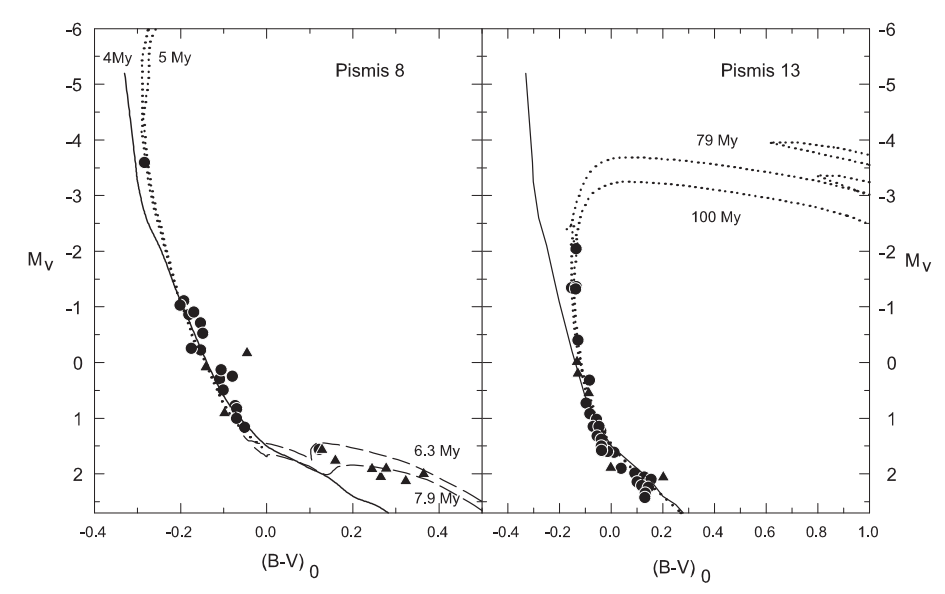

Fig. 9. The corrected color-magnitude diagrams. The Schmidt-Kaler ZAMS (1982) is shown to show the fitting quality. The age is indicated for each isochrone from Girardi et al. (2000) - dotted lines. The pre-main sequence isochrones from Bernasconi \& Maeder (1996) - long-dashed line - and the age are shown superposed onto the Pismis 8 stars.

\subsection{Ages}

The estimation of ages was done by means of a set of isochrones curves obtained from evolutionary models developed by Girardi et al. (2000) that were computed with mass loss and overshooting. Ages listed in Table 8 come from the best isochrone fittings that are shown in Fig. 9. Another possible way of estimating the age of a cluster is look to for the the location of the blue and red "turn-off" points as given by Meynet et al. (1993). This latter procedure was also applied in both clusters as discussed in the following section.

\subsection{Luminosity and mass functions}

The luminosity function, LF, of a star sample is defined as the number of stars found per luminosity bin. This estimate was done in both clusters at $\Delta V=1 \mathrm{mag}$ intervals and the results are shown in Table 6. But even more important, in terms of the cluster formation and evolution, is the estimate of the cluster mass spectrum that describes the distribution of stars over the full mass range. In particular, if such a description refers to the moment of the cluster formation, it is called the initial mass function (IMF). Assuming a power law (see Salpeter 1955), the slope of the mass spectrum is given by

$x=-\log (N / \Delta(\log \mathcal{M})) / \log \mathcal{M}$,

where $N / \Delta(\log \mathcal{M})$ is the number of stars per logarithmic mass interval.

For the estimation of the mass spectra of the clusters, we first transformed the corrected data of all the member stars from the $\left(M_{V}\right.$ vs. $\left.(B-V)_{0}\right)$ plane to the $\left(\log L / L_{\odot}\right.$ vs. $\left.\log T_{\text {eff }}\right)$ plane using bolometric corrections taken from Schmidt-Kaler (1982). Then, the ZAMS mass of each star was obtained using an interpolation procedure between the two closest evolutionary tracks of Schaller et al. (1992) exactly as done in Baume et al. (2003). A detail of the mass spectra of the clusters is given in Table 7. Figure 10 shows the mass spectra and the respective fittings computed from unweighted least squares fittings.
Table 6. Cluster luminosity functions. The number of stars per luminosity bin is indicated for each cluster.

\begin{tabular}{rrr}
\hline \hline$\Delta M_{V}$ & Pismis 8 & Pismis 13 \\
\hline$-4 \cdots-3$ & 1 & \\
$-3 \cdots-2$ & 0 & 1 \\
$-2 \cdots-1$ & 2 & 3 \\
$-1 \cdots 0$ & 6 & 3 \\
$0 \cdots 1$ & 7 & 8 \\
$1 \cdots 2$ & 9 & 17 \\
$2 \cdots 3$ & 4 & 2 \\
\hline
\end{tabular}

Table 7. The mass spectra of the clusters.

\begin{tabular}{rrrr}
\hline \hline \multicolumn{2}{c}{ Pismis 8} & \multicolumn{2}{c}{ Pismis 13 } \\
\hline$\overline{\mathcal{M}}$ & $N / \Delta \log (\mathcal{M})$ & $\overline{\mathcal{M}}$ & $N / \Delta \log (\mathcal{M})$ \\
\hline 1.80 & 60 & 1.87 & 10.31 \\
2.15 & 50 & 2.08 & 154.78 \\
2.88 & 40 & 2.51 & 82.55 \\
4.46 & 45 & 3.14 & 41.28 \\
13.33 & 2.50 & 4.06 & 41.28 \\
& & 4.96 & 20.64 \\
\hline
\end{tabular}

\section{Individual results}

\subsection{Pismis 8}

Pismis 8 was studied by Vogt \& Moffat (1973) by means of $U B V$ photoelectric photometry finding a distance of $1.42 \mathrm{kpc}$ and $E_{B-V}=0.74$. FitzGerald et al. (1979a) also performed $U B V$ photoelectric and photographic photometry finding $1.6 \pm$ $0.25 \mathrm{kpc}$ and $E_{B-V}=0.76 \pm 0.10$ for distance and mean color excess respectively. Only spectroscopy of the brightest cluster star is reported in FitzGerald et al. (1979a,b) for which a spectral classification of B5 $\mathrm{V}$ n and B3 V was given in the respective papers. 


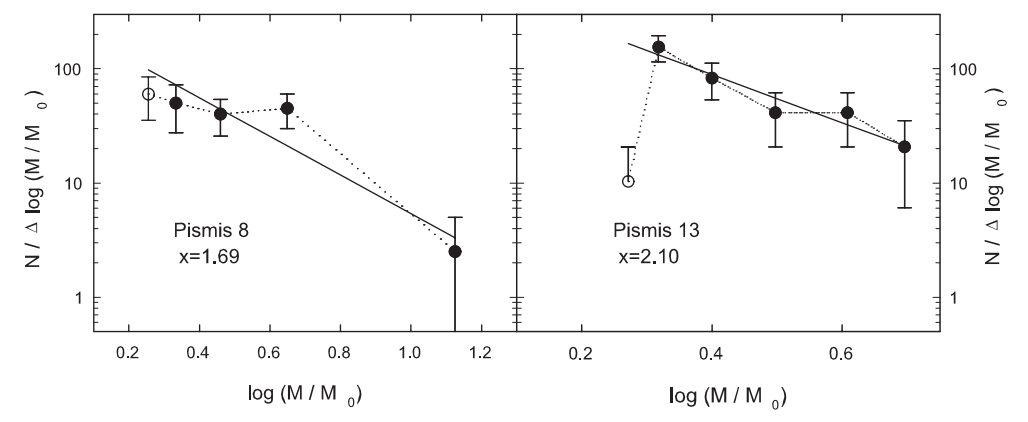

Fig. 10. The mass function of the two clusters. Bars denote the $\sqrt{N} /\left(\Delta \log \left(\mathcal{M} / \mathcal{M}_{\odot}\right)\right)$. Open circles are points not used in the fitting.

Table 8. Properties derived for Pismis 8 and Pismis 13.

\begin{tabular}{lccccccc}
\hline \hline Name & $\alpha_{2000}[\mathrm{~h}: \mathrm{m}: \mathrm{s}], \delta_{2000}[\mathrm{~d}: \mathrm{m}: \mathrm{s}]$ & $l\left[^{\circ}\right], \mathrm{b}\left[^{\circ}\right]$ & Radius $\left[{ }^{\prime}\right]$ & $E_{B-V}$ & $\mathrm{D}[\mathrm{pc}]$ & Age $[\mathrm{Myr}]$ & $x$ \\
\hline Pismis 8 & $08: 41: 36,-46: 16: 00$ & $265.08,-2.63$ & 2.5 & 0.79 & 1995 & $\sim 7$ & $1.69 \pm 0.45$ \\
Pismis 13 & $09: 22: 07,-51: 06: 00$ & $273.15,-0.78$ & 3.0 & 0.61 & 2750 & $79-100$ & $2.10 \pm 0.35$ \\
\hline
\end{tabular}

Note: $\alpha$ and $\delta$ are coordinates of the cluster center as given in Dias et al. (2002).

Figure 3 shows that the stellar density profile of Pismis 8 merges into the background at $2.5^{\prime}$ distance from the cluster center. Therefore, at this angular radius, our photometry includes almost all the cluster area.

Regarding the strong scatter in the upper main sequence (Figs. 4-7) the evidence favors that it is produced by differential reddening as Pismis 8 is seen in the sky projected against a dust lane probably connected to the Gum nebula (RCW 38). Since all across the cluster, dense dust clouds and traces of emission gas are clearly observable, the strong scatter in the upper main sequence of this cluster is the result of a combined effect of dust in front of it plus additional intra-cluster dust. This produces mean color excesses of cluster members of $E_{B-V}=0.79 \pm 0.10$ (s.d) and $E_{U-B}=0.60 \pm 0.08$ (s.d). The mean color excess derived for Pismis 8 is larger than the one quoted by Fitzgerald et al. (1979) probably because more cluster stars have been detected which yields a better estimate of the cluster reddening.

On the other hand, we classified the brightest star, No. 1, as B1V being then of a spectral type earlier than two previous classifications given by FitzGerald et al. (1979a,b) as said above. More remarkable is that this new spectral type produces better agreement with the absolute magnitude derived later on in this article.

The spectral classification of stars No. 14 and 17 is doutful as their spectra have a low signal-to-noise ratio. Despite this we can identify some peculiar features in their spectra such as a weak central emission line $\mathrm{H}_{\beta}$ in star No. 17 (see Table 5 and notes for details).

The reddening law value $\left[A_{V} / E_{B-V}=R\right]$ towards Pismis 8 is shown in Fig. 8. Certainly, the coincidence of the bulk of our data with the reddening line that represents $E_{V-I} / E_{B-V}=1.244$ for normal absorbing material (Dean et al. 1978) suggests that a value of $R=3.1$ holds quite well in this region. Therefore, to obtain corrected magnitudes we applied the usual expression $V_{0}=V-3.1 E_{B-V}$.
The star No. 12 was considered as a probable member; its position in Fig. $9\left((B-V)_{0}=-0.05\right.$ and $\left.M_{V}=-0.17\right)$ suggests that it might be a binary star.

In terms of the cluster distance, the best fit of SchmidtKaler's (1982) ZAMS was achieved for a distance modulus of $V_{0}-M_{V}=11.5 \pm 0.2$ (error from eye inspection), corresponding to a distance of $d=1995 \pm 184 \mathrm{pc}$. This new distance modulus evaluation yielded a value almost half a magnitude larger than the early values of $V_{0}-M_{V}=11.0$ given by Fitzgerald et al. (1979a). We attribute the difference to a better ZAMS fit as more stars defining the faint cluster main sequence are involved in our determination.

We found, on the other hand, that the age of the cluster is about 5-6 Myr, as shown in Fig. 9, a value quite consistent with the "blue turn-off" dating given by Meynet et al. (1993) that yields 7 Myr. So, the cluster is not older than 7 Myr.

When observing the final appearance of the CMD of Pismis 8 in Fig. 9, attention is drawn to the presence of a number of probable cluster members above the ZAMS for $1.5<M_{V}<2.2$ that resemble pre-main sequence stars. If our assumption on the membership of these stars is correct then they would be indicating that the process of star formation has been active until recently in this cluster. Additionally, the age deduced for these stars using the pre main sequence star isochrones of Bernasconi \& Maeder (1996) is approximately $7 \mathrm{My}$. So, it seems that the star formation in this cluster was almost simultaneous for the entire mass range.

Details of the luminosity and mass functions can be found in Tables 6 and 7 and Fig. 10 respectively. The resulting value of the mass function slope of Pismis 8 is $x=1.69 \pm 0.45$, in the mass range from 14 to $1.5 \mathcal{M}_{\odot}$ approximately. Despite unavoidable uncertainties very often found in this type of estimate, the slope value lies between the normal values for IMF slopes quoted by Scalo (1998).

An interesting feature in Pismis 8 is the presence of a gap along the main sequence as pointed out in Fig. 5. Although more details on this kind of feature can be found in 
Giorgi et al. (2002), the current explanations for gaps range from the onset of convection in the stellar envelope (BöhmVitense \& Canterna 1974) to peculiarities in the Balmer jump and Balmer lines as suggested by Mermillod (1976). On the other hand, Ulrich $(1971 \mathrm{a}, \mathrm{b})$ has proposed that gaps in some open cluster might be produced by the onset of ${ }^{3} \mathrm{He}$ isotopes that halts the gravitational contraction 1 or 2 mag above the main sequence.

To quantify the reliability of the gap we follow the Scalo (1986) reasoning assuming that the masses of the consecutively-formed stars are independent of each other (see Giorgi et al. 2002). This way, the probability of finding a gap between $m_{1}=2.02 \mathcal{M}_{\odot}$ and $m_{2}=2.40 \mathcal{M}_{\odot}$ - the stellar masses in the lower and upper sides of the gap in Pismis 8 respectively - with $N=19$ and $x=1.69$ (the number of stars above the upper gap limit and the slope of the cluster mass function respectively) produced by chance is $P_{\text {gap }} \approx 3.9 \times 10^{-3}$.

The brightest cluster star (CD-45 4411) has been considered a "blue straggler" of class 3 with spectral type B3V in the Ahumada \& Lapasset (1995) catalogue. Indeed, the star position $\approx 2$ mag above the second cluster star makes it an intriguing object but, as stated by Ahumada \& Lapasset and Jaschek \& Jaschek (1987), the definition of these objects - above the cluster "turn-off" - is not clear at all. Since the spectral type of this star is B1V and the cluster age we have derived is 5-7 Myr instead of $30 \mathrm{Myr}$ (adopted by Ahumada \& Lapasset), we see that the position of this object along the cluster main sequence is completely congruent with the cluster age. As for its location above the second brightest star, the possibility it might be a binary star should not be ruled out. But most important, this star is a suspected intrinsic variable (Kharchenko 2001). The confirmation of its variability is, therefore, a good test to clarify its true nature.

\subsection{Pismis 13}

$U B V$ photoelectric photometry was made for four stars in Pismis 13 by Vogt \& Moffat (1973) who found $E_{B-V}=0.63$ and a distance of $1.16 \mathrm{kpc}$. Clariá (1979) extended the Vogt \& Moffat work performing $U B V$ photoelectric photometry for 23 stars brighter than $V=16$. He found nine cluster members with $E_{B-V}=0.66$ that situated Pismis 13 at a distance of $2.6 \mathrm{kpc}$. Since the disagreement about the cluster distance in these two papers is very noticeable, another data set is clearly needed to address this question.

Figure 3 shows that the stellar density profile merges into the background level at a distance of approximately $3^{\prime}$. Our observations do not cover the entire cluster, thus the conclusions obtained here are only concerned with the central part of the cluster. Figures 4-7 show that differential reddening is not as strong as in Pismis 8. Although no obvious dust traces are seen across the cluster surface, the inspection of the different DSS plates suggests not only that a dust cloud covers the entire region of the cluster but also that some dust is located inside the cluster itself. Given that several foreground stars are as affected by reddening as Pismis 13 members, the foreground dust cloud could be close to the cluster itself.
The upper main sequence of Pismis 13 appears strongly contaminated by foreground stars. Fortunately, the $U-B$ index is a powerful tool to easily segregate members from non-members as seen in Fig. 6 where foreground stars move redwards off the cluster main sequence, confirming their foreground nature. The computation of the mean color excesses of cluster members yields $E_{B-V}=0.61 \pm 0.055$ (s.d) and $E_{U-B}=0.46 \pm 0.12$ (s.d) that is, of the same order as the previous values determined by Clariá. Carraro \& Munari (2004) determined a mean color excess of $E_{B-V}=0.68$ which was derived from their $B V I$ photometry and not using the $U-B$ index.

The membership of the brightest star cluster obtained from the photometric analysis is reinforced by the spectral classification that we have carried out. Notwithstanding, the four brightest cluster members (stars Nos. 1, 2, 4 and 5) present peculiarities in their spectra. Figure 2 shows that the HeI lines in their spectra are like those in late B- and evolved types, but the CaII and HI lines indicate that they can be early B main sequence stars. For these stars we found no agreement between their spectral types and their colors and magnitudes derived from photometry; a similar behavior is shown by the stars in the Upper Scorpius Complex as discussed by Garrison (1967), and in the Alpha Persei cluster (Morgan et al. 1971).

On the other hand Fig. 8 shows that, as in Pismis 8, the reddening law towards Pismis 13 is normal despite the presence of moderate internal reddening. Under this condition, we computed the absorption-corrected magnitudes adopting $V_{0}=V-3.1 E_{B-V}$.

In relation to the cluster distance, the best fit of the Schmith-Kaler (1982) ZAMS onto the de-reddened main sequence was achieved for a distance modulus of $V_{0}-M_{V}=$ $12.2 \pm 0.2$ including probable cluster members down to $V=16.5 \mathrm{mag}$. The modulus corresponds to a distance of $d=2750 \pm 250 \mathrm{pc}$ in very good agreement with the one determined by Clariá. This distance yields absolute magnitudes for the four brightest cluster members (see Table 5) that are in good agreement with the mean magnitudes expected for stars of this type (Schmidt-Kaler 1982). In this context, the explanation for such a low distance modulus estimation made by Vogt \& Moffat (1973) should be found in the fact that they used few stars and that when fitting the ZAMS they ignored the fact that the brightest cluster stars were all of evolved type. The Carraro \& Munari (2004) study yields a distance of $2.6 \mathrm{kpc}$, in excellent agreement with our determination.

Inspecting Fig. 9, the age of the cluster turns out to be between approximately 79 and 100 Myr. Again, we confirm the good agreement with the "blue turn-off" from Meynet et al. (1993) of 100 My. However, the Carraro \& Munari (2004) age for this cluster is $200 \mathrm{Myr}$. Such a strong difference may have originated partially from the different mean reddenings and, at some extent, in the fact that Carraro \& Munari (2004) did not reject a large number of foreground objects located at the brighter end of the main sequence, as we did.

The details of luminosity and mass functions of Pismis 13, included in Tables 6 and 7, show that cluster star masses are in the range from 5 to $2 \mathcal{M}_{\odot}$. Since this is a too short mass range, the mass function and the slope of the cluster mass spectrum, $x=2.10 \pm 0.35$, are both rather uncertain. Therefore our 
result concerning this cluster mass spectrum should be taken as a preliminary one. Apart from obvious effects (described in Baume et al. 2003) that usually affect the estimation of the IMF in a cluster, we judge that the contamination of field interlopers among the less massive stars may have been very large.

\section{Conclusions}

Pismis 8 and Pismis 13 are two poorly populated open clusters located at distances of 2000 and 2750 pc from the Sun respectively. Combining their distances with the galactic coordinates, they belong to the local spiral arm: Pismis 8 is located along the axis of the local arm as seen from the Sun while Pismis 13 is placed farther at the internal edge of the same structure. Like NGC 2580 (Baume et al. 2004), Pismis 8 and Pismis 13 are spiral tracers with ages of 10 and 100 Myr respectively. Their particular position in the Galaxy explains the high reddening these clusters undergo as the starlight passes through dust between the objects and the Sun. The absorption of these two clusters is in agreement with the absorption maps from Neckel \& Klare (1980), although somewhat larger values are exhibited by the clusters. From Fig. 2, we see that the background star density level in Pismis 8 and 13 reveals how strong the absorption is in the places where they are located, the background density for Pismis 8 being almost half of the one found in Pismis 13. No connection to $\mathrm{H} \alpha$ sources as reported by Georgelin \& Georgelin (1976) has been found for these clusters.

The individual IMF slopes, are larger than expected from a Salpeter (1955) law, 1.69 for Pismis 8 and 2.10 for Pismis 13. These high slope values can arise from a bad subtraction of field stars at faint magnitudes or from uncertainties in the fitting procedure throughout a short mass range (like in Pismis 13). The influence on the computation coming from uncertainties in the star numbers of the less massive bins was overcome by not including them in the fitting. Despite this the values of the slope, 1.69 and 2.10, are still within the slope range indicated by Scalo (1998) for the stellar mass distribution $1<\mathcal{M} / \mathcal{M}_{\odot}<$ $10,1.7 \pm 0.5$.

This article is partially based in the Digitized Sky Survey that was produced at the Space Telescope Science Institute under US government grant NAGW-2166. Original plate material is copyright the Royal Observatory Edinburgh and the Anglo-Australian Observatory.

This publication makes use of data products from the Two Micron All Sky Survey, which is a joint project of the University of Massachusetts and the Infrared Processing and Analysis Center/California Institute of Technology, funded by the National Aeronautics and Space Administration and the National Science Foundation.

This research has made use of the SIMBAD database, operated at $C D S$, Strasbourg, France and of WEBDA, an open cluster data base developed by Jean-Claude Mermilliod.
Acknowledgements. We want to acknowledge the useful comments from Dr. V. Niemela for the treatment and interpretation of the spectroscopic material. We also acknowledge the kind collaboration of the CASLEO staff and Bob Garrison for the allocation of telescope time at UTSO.

\section{References}

Ahumada, J., \& Lapasset, E. 1995, A\&AS, 109, 375

Baume, G., Vázquez, R. A., Carraro, G., \& Feinstein, A. 2003, A\&A, 402, 549

Baume, G., Moitinho, A., Giorgi, E. E., Carraro, G., \& Vázquez, R. A. 2004, A\&A, 417, 961

Bernasconi, P. A., \& Maeder, A. 1996, A\&A, 307, 829

Böhm-Vitense, E., \& Canterna, R. 1974, ApJ, 194, 629

Carraro, G., \& Munari, U. 2004, MNRAS, 347, 625

Clariá, J. J. 1979, The observatory, 99, 202

Cousins, A. W. J. 1978, MNSSA, 37, 62

Dean, J. F., Warren, P. R., \& Cousins, A. W. J. 1978, MNRAS, 183, 569

Dias, W. S., Alessi, B. S., Moitinho, A., \& Lepine, J. R. D. 2002, A\&A, 389, 871

FitzGerald, M. P., Luiken, M., Maitzen, H. M., \& Moffat, A. F. J. 1979a, A\&AS, 37, 345

FitzGerald, M. P., Boudreault, R., Fich, M., \& Luiken, M. 1979b, A\&AS, 37, 351

Garrison, R. F. 1967, ApJ, 147, 1003

Garrison, R. F. 1973, IAU Symp., 50, 13

Georgelin, Y. M., \& Georgelin, Y. P. 1976, A\&A, 49, 57

Giorgi, E. E., Vázquez, R. A., Baume, G. L., Seggewiss, W., \& Will, J.-C. 2002, A\&A, 381, 884

Girardi, L., Bressan, A., Bertelli, G., \& Chiosi, C. 2000, A\&AS, 141, 371

Grotues, H.-G., \& Gocherman, J. 1992, The Messenger, 68, 43

Jaschek, C., \& Jaschek, M. 1987, in The Classification of The Stars (Cambridge Univ. Press)

Kharchenko, N. V. 2001, Kinematika Fiz. Nebesn. Tel., 17, 409

Mermilliod, J.-C. 1976, A\&A, 53, 289

Meynet, G., Mermilliod, J.-C., \& Maeder, A. 1993, A\&AS, 98, 477

Moitinho, A. 2001, A\&A, 370, 436

Morgan, W. W., Hiltner, W. A., \& Garrison, R. F. 1971, AJ, 76, 3

Neckel, Th., \& Klare, G. 1980, A\&AS, 42, 251

Salpeter, E. E. 1955, ApJ, 121, 161

Scalo, J. M. 1986, Fund. Cosm. Phys., 11, 1

Schaller, G., Schaerer, D., Meynet, G., \& Maeder, A. 1992, A\&AS, 96, 269

Schmidt-Kaler, Th. 1982, in Landolt-Börnstein, N.S., Group VI, Vol. 2b, ed. K. Schaifer, \& H.-H. Voigt (Berlin: Springer), 1

Snow, T. P., Zukowski, D., \& Massey, P. 2002, ApJ, 578, 877

Stetson, P. B. 1987, PASP, 99, 191

Tapia, M., Roth, M., Vázquez, R. A., \& Feinstein, A. 2003, MNRAS, 339, 44

Ulrich, R. K. 1971a, ApJ, 165, L95

Ulrich, R. K. 1971b, ApJ, 168, 57

Vázquez, R. A., \& Feinstein, A. 1990, A\&AS, 86, 209

Vázquez, R. A., \& Feinstein, A. 1991, A\&AS, 90, 317

Vázquez, R. A., Baume, G., Feinstein, A., \& Prado, P. 1994, A\&AS, 106,339

Vogt, N., \& Moffat, A. F. 1973, A\&AS, 9, 97 